\title{
A low-cost aerometric sensor system for sport aviation
}

\author{
Jan Auersvald, Karel Draxler, Martin Šipoš *
}

\begin{abstract}
This paper deals with the concept, the design and an experimental prototype of a low-cost aerometric sensor system for sport aviation. The performance of an experimental prototype of the sensor system is compared with the performance of a standard system. The possible further development of the system is also mentioned. The system presented here originates from and extends earlier basic work on an aerometric system for general aviation. The extension implements and focuses general designs from the earlier system for real use of the current system in the category of sport airplanes and gliders. The objective of the system is to reduce the cost of aircraft instrumental equipment, and to provide improved measurement performance of the aerometric system using an innovative approach of altitude measurements. In the experimental system, altitude is still determined based on the classic barometric principle. However, for measurements of atmospheric pressure, the differential pressure sensor is used primarily in combination with a low-cost absolute pressure sensor, which also measures in a differential mode.

K e y w o r d s: aerometric system, differential pressure sensor, sport aviation, altitude measurements, differential pressure
\end{abstract} measurements

\section{Introduction}

Since the beginnings of aviation, airplanes and flying devices have been equipped with instrumental equipment for monitoring the state and the course of the flight. The altimeter is one of the oldest and most basic components of this equipment. It monitors the height of the aircraft $h[2]$. In the area of commercial and military aviation, altimeters were originally standalone mechanical instruments, but they have gradually been developed into the form of an integrated electronic sensor unit, known as the aerometric system [2].

There is a different situation in sport aviation. A survey carried out by the author of this paper revealed that most sport aircraft (more than $80 \%$ in the Czech Republic) are currently equipped with classic standalone instruments. This is primarily due to the cost of modern aerometric systems, which can be as high as twice the cost of classic instruments, and it can amount to $10-20 \%$ of the overall cost of the airplane [3-5]. In addition, the accuracy of standard aerometric systems is still able to only comply with the basic requirements for measurement accuracy in civil aviation (6.1 $\mathrm{m}$ at sea level) [3-5]. The sensitivity of these systems is from $20-50 \mathrm{~cm}$ [3-5], which limits their usability for sensitive determining the derived vertical speed value that is required for gliders in soaring flight. Efforts have therefore been made in recent times to reduce the cost of these systems and to improve their performance. The motivation for the work presented here was to design and develop a low-cost aerometric sensor system for use in sport aviation.

\section{Barometric altitude measurements}

Except in special cases, measurements of altitude $h$, $i e$ the vertical distance from a given reference level, are performed by a barometric method [2]. This method is based on the known course of the dependency of a change in atmospheric pressure on a change in altitude, which can be described according to

$$
h=\frac{T_{0}}{\tau}\left[\left(\frac{p(0)}{p(h)}\right)^{R \tau}-1\right] .
$$

where $h$ is altitude, $p(0)$ is the absolute pressure of the atmosphere in a place at the reference level (standardly, at mean sea level $1013.25 \mathrm{hPa}), p(h)$ is the measured absolute atmospheric pressure by a pressure sensor at a given altitude $h$ (the altitude above mean sea level $h_{M S L}$, if $\left.p(0)=1013.25 \mathrm{hPa}\right), T_{0}=288.15 \mathrm{~K}$, $\tau=6.5 \times 10^{-3} \mathrm{~K} / \mathrm{m}, R=29.271 \mathrm{~m} / \mathrm{K}$ (according to the International Standard Atmosphere (ISA)).

Based on this dependency, it is possible to translate an altitude measurement into a change in atmospheric pressure measurement with the use of a pressure sensor. Standard systems measure the absolute atmospheric pressure value with the use an absolute pressure sensor [2]. To calculate the altitude, standard systems use equation (1).

\section{Application of a differential pressure sensor}

Compared to standard systems, a differential pressure sensor was used to measure changes in atmospheric pressure in this design based on discovered findings from [1].

* Department of Measurement, Faculty of Electrical Engineering, Czech Technical University in Prague, Technická 2, Prague, 166 27, Czech Republic, auersjan@fel.cvut.cz,draxler@fel.cvut.cz, martin.sipos@fel.cvut.cz

DOI: 10.2478/jee-2019-0059, Print (till 2015) ISSN 1335-3632, On-line ISSN 1339-309X 


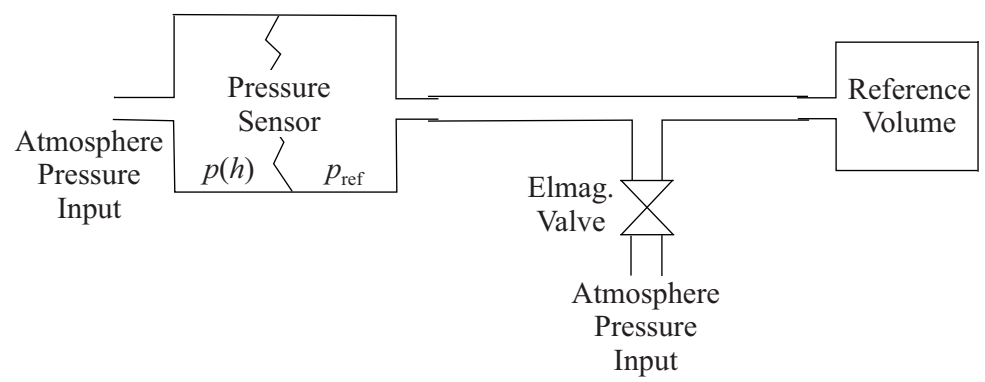

Fig. 1. Differential press.sensor equipped with an auxiliary pressure circuit [1]

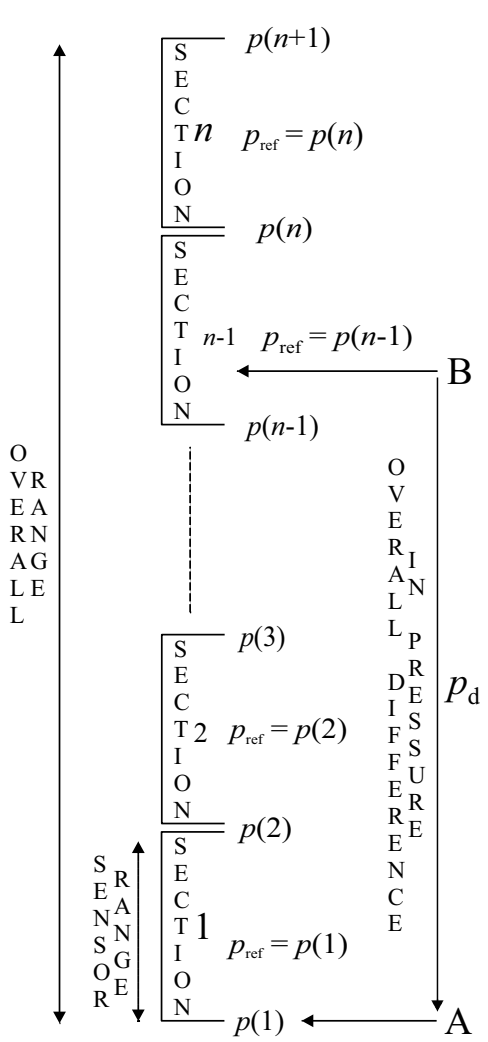

Fig. 2. Schematic diagram of the measurement sections

Although a differential pressure sensor is not able to measure an absolute air pressure value [6,7], it was possible to use it provided that an auxiliary pneumatic circuit, the so-called reference volume (see Fig. 1), was connected to the sensor [8]. This pneumatic circuit brings air at the reference atmospheric pressure $p_{\text {ref }}$ from a known initial place (eg a starting point) into one of the sensor chambers, and it subsequently encloses the air in the chamber.

If air at atmospheric pressure $p(h)$ from the actual place of a measurement is introduced into the second chamber of the sensor, the height above the initial place $h_{d}$ can be expressed from the measured difference in pressure $p_{d g}$ as

$$
h_{d}=\frac{T_{0}}{\tau}\left[\left(\frac{p_{\text {ref }}}{p_{d g}+p_{\text {ref }}}\right)^{R \tau}-1\right]
$$

where $h$ is the altitude of the place of the measurement, $h_{\text {ref }}$ is the altitude of the initial place (assuming the same reference level as for $h), h_{d}=\left(h-h_{\text {ref }}\right)$ is the height above the initial place, $p(h)$ is the absolute pressure of the atmosphere at altitude $h, p_{\text {ref }}$ is the reference absolute pressure of the atmosphere from the initial place at altitude $h_{\text {ref }}, p_{\text {dg }}=\left(p(h)-p_{\text {ref }}\right)$ is the difference between the pressures measured by the differential pressure sensor at height $h_{\mathrm{d}}, T_{0}=288.15 \mathrm{~K}, \tau=6.5 \times 10^{-3} \mathrm{~K} / \mathrm{m}$, $R=29.271 \mathrm{~m} / \mathrm{K}$ (according to the International Standard Atmosphere (ISA)).

If the value for an altitude above mean sea level $h_{\text {refMSL }}$ of the initial place at pressure $p_{\text {ref }}$ is available, it is possible to determine the altitude above mean sea level of measurement place $h_{\mathrm{MSL}}$ by

$$
h_{\mathrm{MSL}}=h_{\mathrm{d}}+h_{\mathrm{refMSL}}
$$

where $h_{\mathrm{MSL}}$ is the altitude above mean sea level of the place of measurement, $h_{\mathrm{d}}$ is the height above the initial place, $h_{\text {refMSL }}$ is the altitude above mean sea level of the initial place.

An advantage of the differential sensor is that its range can be chosen ( $e g \pm 100 \mathrm{hPa}$ in sport aviation), and a differential sensor can operate on multiple smaller measurement sections in the aerometric system with the standard range (eg $0-1000 \mathrm{hPa}$ in commercial and military aviation) $[1,6]$.

Moreover, there can be a much greater maximum relative measurement error (a full-scale error) of the differential sensor in comparison to an absolute pressure sensor, while maintaining the same measurement accuracy [8]. For example, a differential sensor with a range of $\pm 100 \mathrm{hPa}$ can have a more than five times higher allowed maximum relative measurement error than the allowed maximum relative error of an absolute pressure sensor with a range of $1100 \mathrm{hPa}$, while maintaining the same measurement accuracy [8].

These decreased demands then allow the use of a much cheaper differential pressure sensor, which costs between one half and one fifth of the price of an absolute sensor, while meeting the same requirements for measurement accuracy. Alternatively, a more precise differential sensor can provide better measurement accuracy than a similarly precise absolute sensor. A further advantage of the use of 


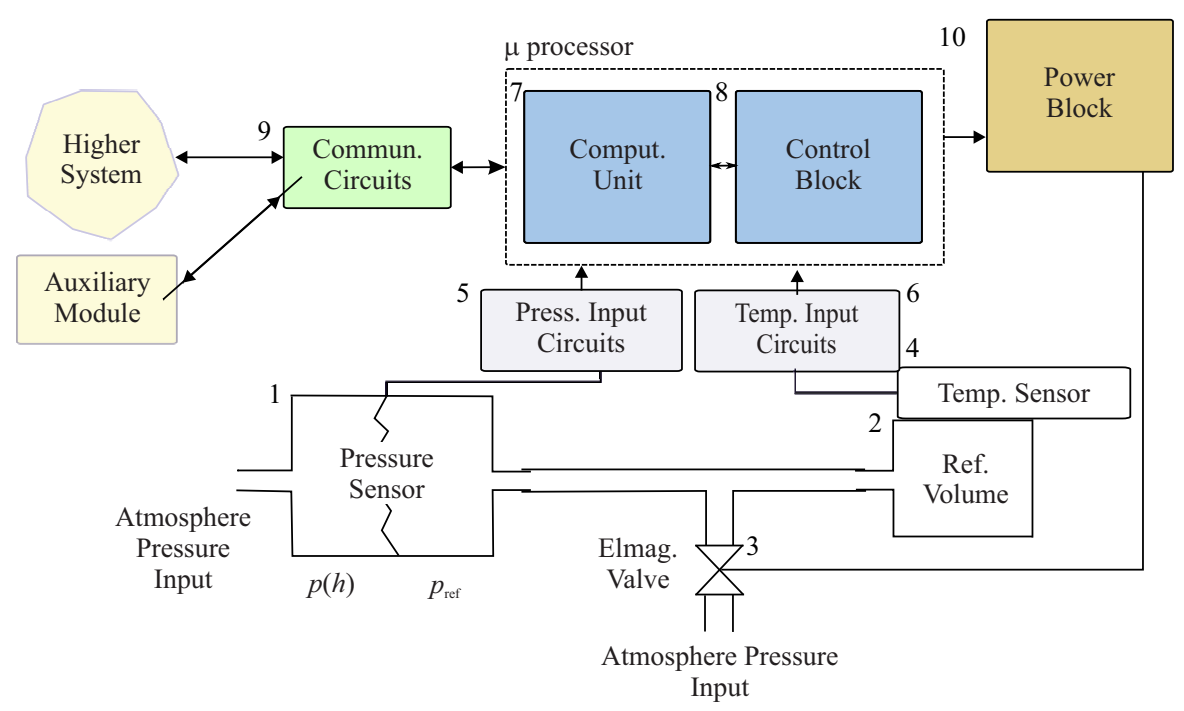

Fig. 3. Block diagram of the main module

a differential sensor with a smaller range is the greater sensitivity and the greater resolution of a differential sensor in comparison to an absolute sensor. This is caused by the diaphragm of the differential sensor, which resists lower mechanical stress and can therefore be thinner and more flexible.

\section{Design of the main module based on the differential pressure sensor}

The main differential module as the core of the aerometric sensor system, expands the basic advantage of the use of a differential sensor with a smaller range by dividing the required measurement range of the system into several measurement sections of the same size, see Fig. 2. The size of these sections $p_{\mathrm{sec}}$ then corresponds to the measurement range of the differential sensor [1].

The overall difference in pressure $p_{\mathrm{d}}$ (for use in further calculations) is then determined as the successive sum of the measured differences in pressure in particular sections, and can be expressed in the case shown in Fig. 2

$$
\begin{aligned}
p_{d}= & p(B)-p(A)=[p(2)-p(1)]+[p(3)-p(2)]+\ldots \\
& +[p(B)-p(n-1)]=p_{d 1}+p_{d 2}+\cdots+p_{d n-1},
\end{aligned}
$$

$p_{d}$ is the overall difference in pressure, $p_{\mathrm{d} 1}, \ldots, p_{\mathrm{d} n-1}$ are the measured differences in pressure in particular sections, $n$ is the number of measurement sections.

If additional errors are not applied, the error in determining the value of the overall difference in pressure $\Delta_{\text {pd }}$ can be expressed according to [9] while using more sections (assuming that all the sections are of the same size and the main module operates over the overall measurement range) as

$$
\begin{aligned}
\Delta_{p_{d}} & =\sqrt{\Delta_{[p(2)-p(1)]}^{2}+\Delta_{[p(3)-p(2)]}^{2}+\ldots+\Delta_{[p(n+1)-p(n)]}^{2}} \\
& =\Delta_{p_{\mathrm{sec}}} \sqrt{n}=\delta_{p_{\mathrm{sec}}} p_{\mathrm{sec}} \sqrt{n}
\end{aligned}
$$

where, $\Delta_{\mathrm{pd}}$ is the maximum error in determining the value of the overall difference in pressure, $\Delta_{[p(2)-p(1)]}=$ $\Delta_{[p(3)-p(2)]}=\cdots=\Delta_{[p(n+1)-p(n)]}=\Delta_{p s e c}$ is the maximum error in determining the difference in pressure in the measurement section using the differential sensor, $\delta_{p s e c}$ is the maximum relative error in determining the difference in pressure in the measurement section using the differential sensor, $p_{s e c}$ is the size of the measurement section, $n$ is the number of measurement sections.

The block diagram of the main differential module is shown in Fig. 3.

The core of the module consists of the differential sensor $\langle 1\rangle$. The first pressure input of the differential sensor is led into the outer atmosphere at pressure $p(h)$. The second pressure input is connected to the reference volume $\langle 2\rangle$, which is formed by a container to keep the air at reference pressure $p_{\text {ref }}$, and an electromagnetic valve $\langle 3\rangle$. The reference volume is equipped with a precise temperature sensor $\langle 4\rangle$. The output of the differential pressure sensor and the output of the temperature sensor are connected to a microprocessor through the input circuits $\langle 5,6\rangle$. The microprocessor provides the function of a computational unit $\langle 7\rangle$ and a control block $\langle 8\rangle$. In order to communicate with a higher system, the main module is equipped with communications circuits $\langle 9\rangle$. The power supply for the main module and the power control of the electromagnetic valve are provided by the power block $\langle 10\rangle$. At the beginning of the measurement, the control block $\langle 8\rangle$ opens the electromagnetic valve $\langle 3\rangle$ at initial place $\mathrm{A}$, see Fig. 2, and fills the reference volume $\langle 2\rangle$ with air at the reference pressure $p_{\text {ref }}=p(1)$ from place A. At the same time, the initial temperature of the air in the reference volume is measured by an accurate temperature sensor $\langle 4\rangle$. The electromagnetic valve is closed. Then, the differential sensor $\langle 1\rangle$ starts to measure the difference $p_{\mathrm{d} 1}$ between the atmospheric pressure $p(h)$ at the current place of measurement and the reference pressure $p_{\text {ref }}$ in first measurement section. At the same time, the current temperature of the air in the reference volume is measured 
by the accurate temperature sensor. The output values of the differential sensor and the accurate temperature sensor are then fed into the computational unit $\langle 7\rangle$ and the control block. The computational unit performs a compensation of the output value of the differential sensor and recalculates the compensated value into the height above the initial place $h_{\mathrm{d}}$, according to (2). The calculated value $h_{\mathrm{d}}$ is then sent to the higher system.

Simultaneously, the control block controls the state of the module. Now when the value of the measured difference in pressures $p_{\mathrm{d} 1}$ approximately reaches the size of the measurement section $p_{\mathrm{sec}}$ (the range of the differential sensor), the control block switches the module into the second measurement section [1]. The "switching" (see Fig. 2) consists of three steps. The first step is to save the last value of the measured difference in pressure $p_{\mathrm{d} 1}$ by the computational unit. The second step is to open the electromagnetic valve and to fill the reference volume with air at pressure $p_{\text {ref }}=p(2)$ from the place of the "switching". The third step is to measure the new initial temperature of the air in the reference volume in order to compensate the output value of the differential sensor in the second section.

The differential module starts to measure again the difference $p_{\mathrm{d} 2}$ in the second measurement section. This difference is added to the last value of the measured difference in pressure $p_{\mathrm{d} 1}$ in the first section by the differential module, according to (4). The overall difference in pressure $p_{\mathrm{d}}$ is recalculated again into the height above the initial place $h_{\mathrm{d}}$, according to (2), with the fact that for the purposes of this (re)calculation, the value $p_{\text {ref }}$ is still $p_{\text {ref }}=p(1)$. The procedure is repeated. Now when the value of the measured difference in pressure $p_{\mathrm{d} 2}$ approximately reaches the size of the measurement section $p_{\text {sec }}$ again, the control block performs the next "switching" into the third measurement section, etc, see Fig. 2.

In the case of "switching" from a higher measurement section to a lower measurement section, the situation is similar, with the distinction that, after the "switching", the actual value of the measured difference between $p(h)$ and $p_{\text {ref }}$ is positive. The actual value is then added to the negative values of the difference in pressure measured in the previous sections.

There is a special situation when the main differential module reaches the highest determined overall difference in pressure $p_{\mathrm{d}}$ (the highest measurement place) and the climb changes to a descent. In this case, the control block switches the module into a lower measurement section at the time when the measured difference in pressure changes from a negative value to a positive value and approximately reaches the size of the measurement section $p_{\text {sec }}$, see Fig. 2.

This allows a much lower number of necessary "switching" of the module, which can be used to increase the accuracy of the module (see below) and to increase the lifetime of the electromagnetic valve.

\section{Compensating the principle disadvantages of the main module}

To ensure that the overall difference in pressure $p_{\mathrm{d}}$ is measured accurately, it was necessary to compensate for two significant disadvantages [1].

The first disadvantage derives from the need to use the reference volume $[1,8]$. Compared with the invariable vacuum, the reference pressure of the air enclosed in the reference volume depends on changes in external conditions, especially changes in temperature [10].

If the air is considered as a real gas, this dependency and the compensation for it is rather complex. In our design, the air was simply considered as an ideal gas, the behavior of which can be described by the general gas equation (the ideal gas law), and only basic compensation was performed. This compensation is performed only on the basis of a measurement of the changes in the air temperature in the reference volume. The change in the reference atmospheric pressure $\Delta p_{\text {ref }}$ caused by a change in temperature can be expressed according to [11], as follows

$$
\Delta p_{\text {ref }}=p_{\text {ref }}\left(\frac{T_{2}-T_{1}}{T_{1}}\right)
$$

where $\Delta p_{\text {ref }}$ is the change in the reference pressure of the atmosphere in the reference volume caused by a change in temperature, $p_{\text {ref }}$ is the initial reference absolute pressure of the atmosphere, $T_{1}$ is the initial temperature of the air at the reference pressure of the atmosphere, $T_{2}$ is the actual temperature of the air at the reference pressure of the atmosphere.

A second disadvantage of the module is the "interruption" of the measurements when it switches between measurement sections [1]. During a fast climb or a fast descent of an airplane in the "switching" phases, this "interruption" of the measurement can cause a large error in determining the overall difference in pressures $p_{\mathrm{d}}$.

In addition, the value of this error has an integrative character, and its behavior depends on the actual maneuver of the airplane at the time of each "switching". Because it is not possible to predict the change in altitude or, more precisely, the corresponding change in the overall difference in pressure that arises during the "switching" [1], it was necessary to design an auxiliary module to suppress a possible error. This auxiliary module determines an "auxiliary value" about the change in the overall difference in pressure that arises when the main module is "switched".

\section{Design of the auxiliary module}

The auxiliary module is based on the use of a lowcost absolute pressure sensor. A block diagram of the auxiliary module is presented in Fig. 4. The absolute sensor $\langle 1\rangle$ is connected to the internal computational unit of the auxiliary module $\langle 3\rangle$ through input circuits $\langle 2\rangle$. The internal computational unit sends final calculations and output values via communications circuits $\langle 4\rangle$ into 


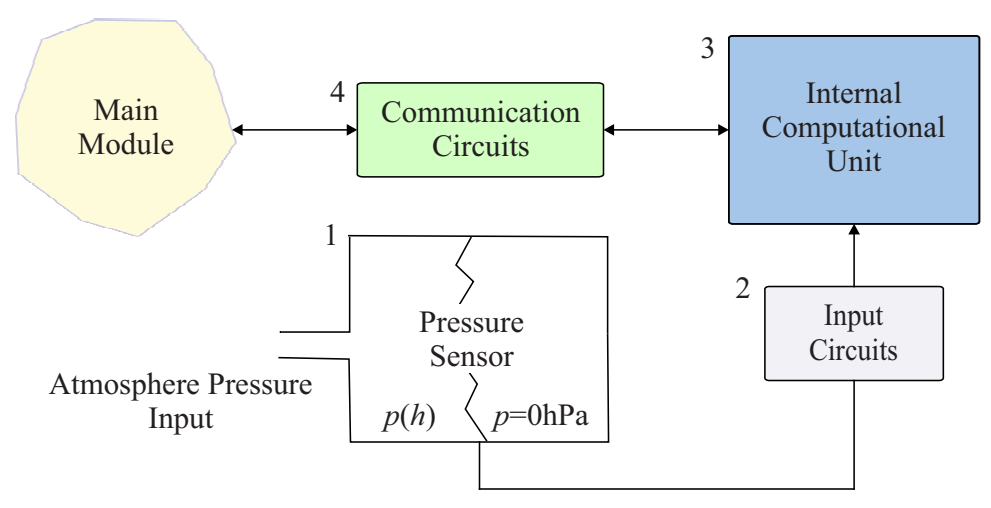

Fig. 4. Block diagram of the auxiliary module [1]

the main module. These last three blocks are integrated into the microprocessor.

The determination of the "auxiliary value" is based on determining the changes (the difference) between a current absolute value and an initial absolute value of the atmospheric pressure. When "switching" of the main module begins, the internal computational unit $\langle 3\rangle$ of the auxiliary module saves the current absolute value of the pressure measured by the absolute pressure sensor $\langle 1\rangle$ as its initial absolute value $p_{\text {ia }}$. When the "switching" of the main module has ended, the internal computational unit of the auxiliary module subtracts the saved initial absolute value $p_{i \text { a }}$ from the current measured absolute value of the pressure $p_{\mathrm{aa}}$. The difference between the absolute atmospheric pressure values $p_{\text {da }}=p_{\text {aa }}-p_{\text {ia }}$ is sent into the main module. The main module adds the "auxiliary value" to the last overall difference in pressures $p_{\mathrm{d}}$ and then starts making measurements on a new measurement section.

This principle reduces a error of the absolute pressure measurements when a low-cost absolute pressure sensor is used. The error is caused partially by the low long-term stability of the offset of the transfer characteristic and the nonlinearity of the transfer characteristic. The effect of low long-term stability is reduced by using differential determination of the "auxiliary value". In addition, a part of the nonlinearity of the transfer characteristic (for a close position of the initial value and the current measured absolute value of the pressure) is also partially suppressed.

All the procedures mentioned here enable a low-cost absolute pressure sensor to determine the "auxiliary value" reliably.

The use of a low-cost absolute sensor then keeps down the cost of the aerometric sensor system. If these procedures had not been developed, it would have been necessary to use an expensive precise absolute pressure sensor for standard aerometric systems. This would have raised the costs, and it would have been necessary to abandon the idea producing a low-cost aerometric sensor system.

Once the auxiliary module is added to the main module, the overall difference in pressures $p_{\mathrm{d}}$ can be expressed by the extension of (4) in the case shown in Fig. 2

$$
\begin{aligned}
p_{d}=p(B)-p(A)=p_{\mathrm{d} 1} & +p_{\mathrm{d} 2}+, \ldots,+p_{\mathrm{d} n-1}+ \\
& +p_{\mathrm{da} 1}+p_{\mathrm{da} 2}+\cdots+p_{\mathrm{da} m},
\end{aligned}
$$

where, $p_{\mathrm{d}}$ is the overall difference in pressure, $p_{\mathrm{d} 1}$, $\ldots, p_{\mathrm{d} n-1}$ are the measured differences in pressure in particular measurement sections, $p_{\text {da } 1} \ldots, p_{\text {dam }}$ are the "auxiliary values" about the change in the overall difference in pressure that arises when the main module is "switched", $n$ is the number of measurement sections, $m$ is the number of "auxiliary values" about the change in the overall difference in pressure that arises when the main module is "switched".

The error in determining the value of the overall difference in pressure $\Delta_{\mathrm{pd}}$ (the auxiliary module is added to the main module for the overall measurement range of the main module) can be expressed according to [9] by extending (5), as

$$
\begin{aligned}
& \Delta_{p_{d}}=\sqrt{\Delta_{p_{\mathrm{sec}}}^{2} n+\Delta_{p_{a v}}^{2} m}= \\
& \sqrt{\Delta_{p_{\mathrm{sec}}}^{2} n+\Delta_{p_{\mathrm{av}}}^{2}(2(n-1)-1)}
\end{aligned}
$$

where $\Delta_{\mathrm{pd}}$ is the maximum error in determining the value of the overall difference in pressure (the auxiliary module is added to the main module), $\Delta_{p_{\mathrm{sec}}}$ is the maximum error in determining the difference in pressure in a measurement section using the differential sensor, $n$ is the number of measurement sections, $\Delta_{p_{\text {av }}}=$ $\max \left(\Delta_{p_{\mathrm{da} 1}}, \Delta_{p_{\mathrm{da} 2}}, \ldots, \Delta_{p_{\mathrm{da} m}}\right)$ is the maximum error in determining the changes (the difference) in the absolute value of the pressure (the "auxiliary value"), (for a change $< \pm 20 \mathrm{hPa}$ in size), using the auxiliary module in the whole range of the measurement, $m=2(n-1)-1$ (assuming an ordinary type of flight) is the number of "auxiliary values" about the change in the overall difference in pressure that arises when the main module is "switched".

\section{Experimental prototype}

The prototype of the main module is based on the Honeywell HSC001PD differential sensor with a measurement 


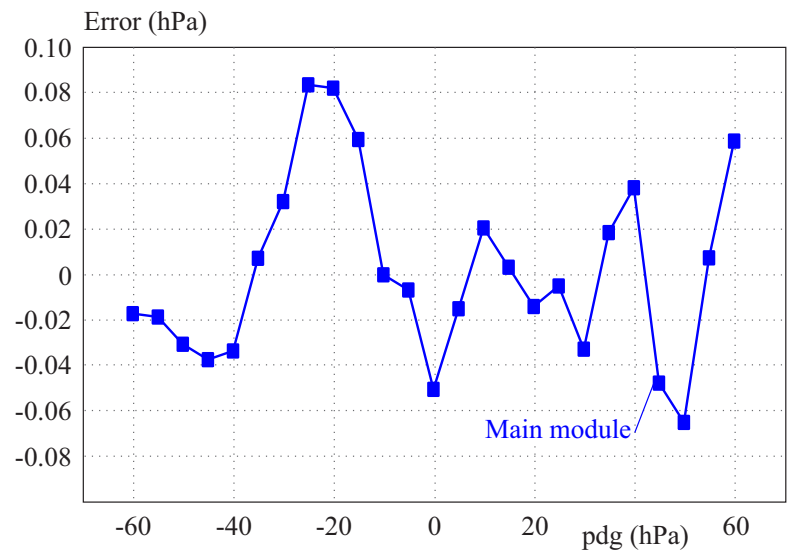

Fig. 5. Error in determining the difference in pressure of the main module

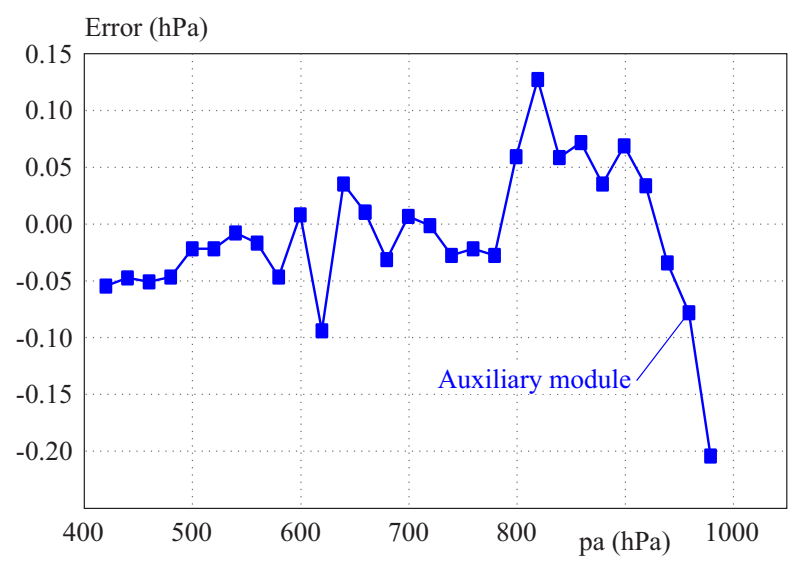

Fig. 6. Error in determining the "auxiliary value" of the auxiliary module

section $p_{\text {sec }}$ (range of the sensor) $\pm 69 \mathrm{hPa}$ in size [12]. A steel container $10 \mathrm{ml}$ in volume and a Fluid Automation Systems 01-341P-00-H0 electromagnetic valve were used for the realization of the reference volume. A Heraeus L1020 precise temperature sensor was used for the temperature measurements in the reference volume container.

The prototype of the auxiliary module is based on the low-cost BMP 180 absolute pressure sensor [13]. The absolute pressure sensor is thermo-regulated at a constant temperature to suppress the temperature errors [14] by the heated PCB. The ATMEGA8 auxiliary module microprocessor controls the heating, reads and processes the absolute pressure sensor values. It also works as a higher system for the main module and gathers all values for evaluation tests.

\section{Evaluation of the experimental prototype}

In the first part of the evaluation, calibrations were performed, and the basic characteristics of the pressure and temperature sensors of both modules were determined. During this part of our work, the prototype of the main module was measured in a single mode only in the first measurement section (in the range of $\pm 69 \mathrm{hPa}$ ), which corresponds to the range of measurements of a height of $\pm 590 \mathrm{~m}$ above the initial place.

In this mode, the prototype of the main module reached the maximum error in determining the difference in pressure in the measurement section $\Delta_{\text {psec }}=0.08 \mathrm{hPa}$ and sensitivity of $0.02 \mathrm{hPa}$. The course of this error of the main module according to a measured difference in pressure is shown in Fig. 5.

The prototype of the auxiliary module was tested for the designed measurements in the range of $500-1040 \mathrm{hPa}$ (220-5500 m). In this range, the prototype can determine the change in the absolute value of the pressure (the "auxiliary value") (for a change $< \pm 20 \mathrm{hPa}$ in size) with a maximum error of $\Delta_{\text {pav }}=0.20 \mathrm{hPa}$. The course of this error of the auxiliary module according to a measured absolute pressure is shown in Fig. 6.

For further tests, an artificial flight of a generic sport airplane was also specified. The model flight consisted of a climb element, in the course of which the airplane steadily climbs for 25 minutes from $0 \mathrm{~m}$ to $2500 \mathrm{~m}$ at a constant vertical speed of $1.6 \mathrm{~m} / \mathrm{s}$. The flight then turns into a descent, in the course of which the airplane descends steadily to $0 \mathrm{~m}$ at the same vertical speed.

On the basis of the results mentioned above, it was possible to calculate the theoretical error in determining the value of the overall difference in pressures of the whole system (ie the auxiliary module is added to the main module) according to (8) for the artificial flight at the end of the first part of the evaluation. The whole system, which operates in five measurement sections (based on an artificial flight, with a range of measurements of a height of $0-2850 \mathrm{~m}$ above the initial place), has a theoretical error of $\Delta_{\mathrm{pd}}=0.56 \mathrm{hPa}$. This error corresponds to the theoretical error in determining the height above the initial place (sea level), according to ISA, $\Delta_{\text {hd }}=4.7 \mathrm{~m}$. The theoretical sensitivity of the whole system corresponds to the sensitivity of the main module, ie $0.02 \mathrm{hPa}$. This corresponds to the change in height above the initial place (sea level), which is $16 \mathrm{~cm}$ (according to the ISA).

These theoretical results represent the ideal case for the operation of the whole system, and they also show the extent to which the real whole system could be improved.

After the first part of the evaluation, the second part of the evaluation was performed. It consisted of a complex laboratory test, which simulated the conditions of the artificial flight for the whole system.

The whole system, running in full operation, was enclosed in a pressure chamber. The pressure in the chamber was controlled by a PACE6000 precise pressure regulator, and the course of the controlled pressure followed the artificial flight. For the whole artificial flight (50 minutes), continuously processed values of the differences in pressure set in the chamber (delivered by the pressure regulator) and the differences in pressure measured in the chamber (delivered by the whole system) was being gathered. 


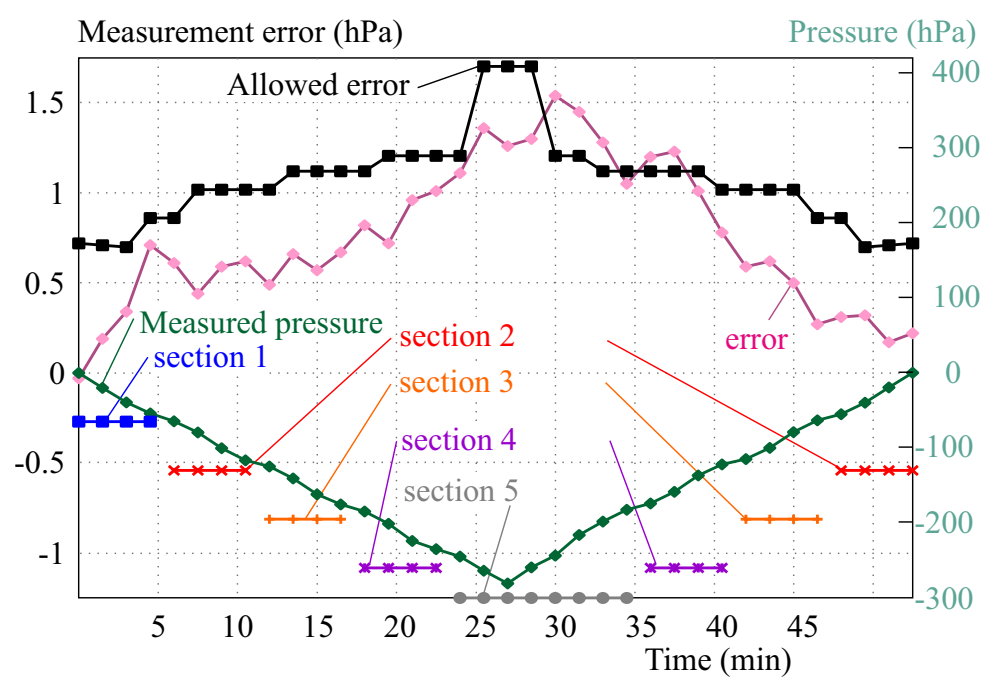

Fig. 7. Results from the laboratory test

The results of the laboratory test are shown in Fig. 7 . The bottom part of the figure shows a record of the measured difference in pressure during the artificial flight. It also indicates in which measurement section the main module was making measurements during the corresponding part of the artificial flight. The upper part of the figure shows a record of the measurement error of the whole system. This was determined as the difference between the set differences in pressure and the measured differences in pressure. The record of the measurement error is accompanied by a representation of the allowed measurement error in the corresponding part of the artificial flight, based on the required accuracy of height measurements in civil aviation [2].

Despite the good theoretical results, the results of the laboratory test show that the whole system is influenced by other systematic and random errors when it is in full operation. The record of the measurement error led to the following findings: The measurement error increases with increasing measured differences in pressure and when higher measurement sections are reached. The measurement error decreases with decreasing measured differences in pressure and with a return to lower measurement sections, as was expected from the theoretical analysis. "Switching" causes an irreversible integrative part of the measurement error. However, this part is sufficiently compensated by the auxiliary module. The asymmetrical course and the shifted maximum of the measurement error to the beginning of the descent part of the model flight are probably caused by unexpected pressure effects in the pneumatic system of the reference volume and the differential pressure sensor during the change from a negative difference to a positive difference in the pressures in this system. These effects will be a topic for further research.

The real error in determining the value of the overall difference in pressure of the whole system (based on the results of the laboratory test) in the first measurement section (take-off and landing operations) was cal- culated to be $\Delta_{\mathrm{pd} 1}=0.71 \mathrm{hPa}$. This error corresponds to the real error in determining the height above the initial place (sea level), according to ISA $\Delta_{\text {hd }}=5.9 \mathrm{~m}$. The real error in determining the value of the overall difference in pressure of the whole system (based on the results of the laboratory test) in whole range was calculated to be $\Delta_{\mathrm{pd}}=1.54 \mathrm{hPa}$. This error corresponds to the real error in determining the height above the initial place (sea level), according to ISA, $\Delta_{\mathrm{hd}}=12.8 \mathrm{~m}$. However, it should be noted that these values of the error in whole range were calculated from the maximum measurement error by the standard procedure. This maximum measurement error occurs only in the highest measurement sections, and it does not represent the behavior of the measurement accuracy of the whole system in whole range, especially in the proximity of the initial place (take-off and landing). This statement can be justified by the calculated errors in the first measurement section, and by the graphical comparison of the measurement error and the allowed measurement error in Fig. 7. The whole system complies with the allowed measurement error, except for the beginning of the descent part of the model flight. However, this exception does not exceed the allowed limit for more than $0.33 \mathrm{hPa}$. The real sensitivity of the whole system also corresponds to the sensitivity of the main module, ie $0.02 \mathrm{hPa}(16 \mathrm{~cm})$.

\section{Conclusions}

According to the evaluation that was performed, the concept, the design, and the experimental prototype of the aerometric sensor system for sport aviation have the prerequisites for meeting the demands for the accuracy of height measurements required in civil aviation [2].

In comparison with the standard systems, our experimental prototype has 1.03 times (3\%) greater accuracy (in the first measurement section) and approximately 2 times $(100 \%)$ better sensitivity, thanks to the use of the 
differential pressure sensor. In addition, according to the theoretical results, there is a potential for up to $30 \%$ improvement of accuracy.

The cost of the experimental prototype of the aerometric sensor system is approximately only $40 \%$ of the average cost of a standard aerometric system based on an absolute pressure sensor [3-5].

This system could therefore be an interesting alternative to standard aerometric systems based on an absolute pressure sensor, especially for gliders, thanks to its sensitivity performance.

The disadvantages of the aerometric sensor system are the more complicated structure, due to the need to use the "reference volume", and the limited lifetime of the system corresponding to the lifetime of the electromagnetic valve. When the aerometric sensor system is used as a standard altimeter, the need for information about the height above mean sea level of the initial place, and also the absolute atmospheric pressure value from the initial place can also be considered as a certain disadvantage from the point of view of the user. However, both values are normally available on each airport.

A further evaluation and improvements to the experimental prototype of the aerometric sensor system is a subject for further development. In particular, an investigation needs to be made into the behavior of the pneumatic system of the reference volume and the differential pressure sensor during full operation, and it is necessary to improve the "switching" procedure and the modules to bring the real measurement error close to the theoretical potential. After these steps, an evaluation of the final aerometric sensor system can potentially be made in real flight tests.

\section{Acknowledgements}

This work was supported by the Grant Agency of the Czech Technical University in Prague, under grant No. SGS19/113/OHK3/2T/13: "Usage of alternative sensors and systems for localization of mobile devices".

\section{REFERENCES}

[1] J. Auersvald and K. Draxler, "Aerometric System for General Aviation", International Conference on Military Technologies (ICMT) 2015, pp. 563-568, 2015.

[2] E. H. J. Pallett Aircraft Instruments \& Integrated Systems, 1st ed. Harlow, UK: Longman Group UK Ltd, pp. vii-viii, 25-29, 161-167, 1992.

[3] TL Elektronic - Light Sport Aircraft, 7 EFIS INTEGRA 2019 [Online] Available:

http://www.tl-elektronic.com/index.php?page=lsa\&p_id=19

[Accessed: 30-Jun-2019].

[4] Dynon Avionics - Flight Instruments - EFIS-D10A Dynonavionics.com, 2019, [Online], Available: https://www.dynonavionics.com/efis-d10a.php. [Accessed: 30-Jun-2019].
[5] Altimeter - Kanardia Kanardia, 2019, [Online], Available: https://www.kanardia.eu/product/altimeter/ [Accessed: 30-Jun-2019].

[6] D. Tandeske, Pressure Sensors Selection Application, 1st ed, New York, NY, USA: Marcel Dekker Inc., pp. 178-187, 198-200, 1991.

[7] J. Vetelino and A. Reghu, "Introduction to Sensors", 1st ed, Boca Raton, FL, USA: CRC Press, 2011.

[8] J. Auersvald, "Measurement of Barometric Altitude for UAV", MS thesis CTU in Prague, 2012.

[9] Team of authors of JCGM, Guide to the Expression of Uncertainty in Measurement, 2008.

10] D. Bolanakis, K. Kotsis, and T. Laopoulos, "Temperature Influence on Differential Barometric Altitude Measurements", 2015 IEEE 8th International Conference on Intelligent Data Acquisition Advanced Computing Systems: Technology Applications (IDAACS) pp. 120-124, 2015, Available: https:// ieeexplore.ieee.org /document/7340711 [Accessed 30 June 2019]

11] W. Kauzmann, "Kinetic Theory of Gases", 1st ed, New York, NY, USA: Dover Publications, 2013, pp. 10-21.

[12] TruStabilityR Silicon Pressure Sensors: HSC Series-High Accuracy, Golden Valley MN, USA: Honeywell International Inc., 2011.

13] BMP 180 Digital Pressure Sensor, Reutlingen Germany: Bosch Sensortec GmbH, 2015.

[14] Z. Jiao, J. Hu, and X. Gong, "Research of Altitude Measurement Method Based on INS Pressure Sensor", 2017 IEEE 2nd Information Technology Networking, Electronic Automation Control Conference (ITNEC), pp, 1684-1687, 2017, Available: https://ieeexplore.ieee.org/document/8285082 [Accessed 30 June 2019].

Received 16 July 2019

Jan Auersvald was awarded his bachelor degree in cybernetics and measurement from the Czech Technical University in Prague (CTU), Prague, Czech Republic in 2010, and his master"s degree in aircraft and space systems from CTU, in 2012. He is currently studying for a $\mathrm{PhD}$ degree in air traffic control at CTU. From 2012 to 2013, he was a Research Assistant with the Laboratory of Aircraft Instrumentation Systems. Since 2013, he has been a Flight Simulator Engineer with the Czech Aviation Training Centre, Prague. His research interests include aerometric systems based on low-cost differential and absolute pressure sensors and other aerometric systems.

Karel Draxler received his master degree in radio engineering from the Czech Technical University in Prague (CTU) in 1963 and completed his PhD studies in 1974. He defended his inaugural dissertation in 1998. He is working at the department of measurement of the Faculty of Electrical Engineering of CTU. Since 1975 he has been involved in the field of aircraft equipment in the low frequency range. He has lectured on Aircraft Instruments and Systems, and led a laboratory investigating this subject at the Faculty of Electrical Engineering.

Martin Šipoš received the Ing degree (MSc equivalent) and the PhD degree from the Faculty of Electrical Engineering (FEE), Czech Technical University (CTU), Prague, Czech Republic, in 2008 and 2015, respectively. He is an Assistant Professor and Researcher with the Department of Measurement, FEE, CTU. His main research interests are in avionics, inertial navigation systems, GNSS, sensors, and their calibration and modeling, and data processing methods. 